\title{
Altersbedingte echte Exfoliation der Linsenkapsel: Ergebnisse der Phakoemulsifikationsoperation
}

\author{
Alex Lap Ki Ng ${ }^{a}$ Marcus M. Marcet ${ }^{\mathrm{a}} \quad$ Jimmy S.M. Lai ${ }^{\mathrm{a}} \quad$ Jane C.C. Yeung ${ }^{\mathrm{b}, \mathrm{c}}$ \\ a Department of Ophthalmology, LKS Faculty of Medicine, The University of Hong Kong, \\ ${ }^{b}$ Department of Ophthalmology, Queen Mary Hospital, and \\ 'Premier Medical Centre, Hong Kong, SAR, China
}

Schlüsselwörter

Echte Exfoliation · Linse · Katarakt · Phakoemulsifikation

\begin{abstract}
Zusammenfassung
Die echte Exfoliation der kristallinen Linse, historisch gesehen ein bei den Glasbläsern beobachtetes Phänomen, beinhaltet eine Aufspaltung oder Abschilferung der Kapsel. Wir haben die Phakoemulsifikationsaufzeichnungen eines einzelnen Chirurgen im Hinblick auf Patienten mit echter Exfoliation der Linsenkapsel überprüft. Die Inzidenz in unserer Serie betrug 2,2\% (6 von 278 Fällen). Das Durchschnittsalter betrug 85 Jahre. Bei allen Patienten verlief
\end{abstract}

die Phakoemulsifikation erfolgreich, was auf die genaue Feststellung der Erkrankung und entsprechende chirurgische Planung zurückzuführen sein könnte. Unsere Erkenntnisse unterstützen die Auffassung, dass echte Exfoliation möglicherweise häufiger mit fortgeschrittenem Alter assoziiert ist als mit Infrarotstrahlung.

(c) 2017 S. Karger GmbH, Freiburg

\section{Einführung}

Die echte Exfoliation der kristallinen Linse beinhaltet eine Aufspaltung oder Abschilferung der Kapsel. Historisch wurde das Phänomen auch als Glasbläserstar bezeichnet aufgrund des bekannten Risikos, die Krankheit durch anhaltende Exposition gegenüber Hitze und Infrarotstrahlung zu verursachen [1]. Zu anderen okularen Zuständen, die mit echter Exfoliation assoziiert sind, zählen Alter, Trauma, Uveitis und Glaukome [2, 3]. Das Bewusstsein für die Verbindung zwischen fortgeschrittenem Alter und echter Exfoliation der Linsenkapsel ist gestiegen. Beispielsweise wurden in einer Studie mithilfe von Transmissionselektronenmikroskopie altersbedingte ultrastrukturelle Veränderungen bei einem Patienten mit echter Exfoliation der Linsenkapsel festgestellt [4].

In der vorliegenden Fallserie berichten wir über 6 Patienten, die sich einer Phakoemulsifikation mit echter Exfoliation der Linsenkapsel unterzogen. Wir erkannten die echte Exfoliation entweder präoperativ an einer aufgerollten und/oder gespaltenen vorderen Kapsel oder intraoperativ anhand des Doppelringzeichens [1]. Alle Behandlungen wurden unter örtlicher Betäubung von demselben Chirurgen (J.C.C.Y.) in einem Zeitraum von 6 Monaten durchgeführt und gehören zu insgesamt 278 Fällen, die im gleichen Zeitraum operiert wurden.

\section{Falldarstellungen}

Die klinischen Merkmale der 6 Patienten sind in Tabelle 1 zusammengefasst. Das Durchschnittsalter betrug 85,0 \pm 7,1 Jahre (Range: 72-92). Keiner der Patienten hatte eine Vorgeschichte mit längerer Infrarotbestrahlung oder Hitzebelastung, Trauma oder Augenentzündung. Bei 3 Patienten (Fälle 1, 5, 6) erfolgte die klinische Diagnose einer echten Exfoliation präoperativ mittels Spaltlampenuntersuchung (Abb. 1). In den Fällen 1 und 6 wurden die vor-

\section{KARGER}

Fax +4976145207 14

information@karger.com

www.karger.com

\section{(c) 2017 S. Karger GmbH, Freiburg}

Accessible online at

www.karger.com/kop
Dr. Jane C.C. Yeung

8/F, Block S, Queen Mary Hospital

102 Pokfulam Road

Hong Kong, SAR (China)

yeung.jane@gmail.com 
deren Kapseln zur histologischen Bestätigung eingeschickt (Abb. 2). Bei den anderen 3 Patienten (Fälle 2, 3, 4) wurde die Erkrankung erst intraoperativ erkannt, und es wurde keine histolo-

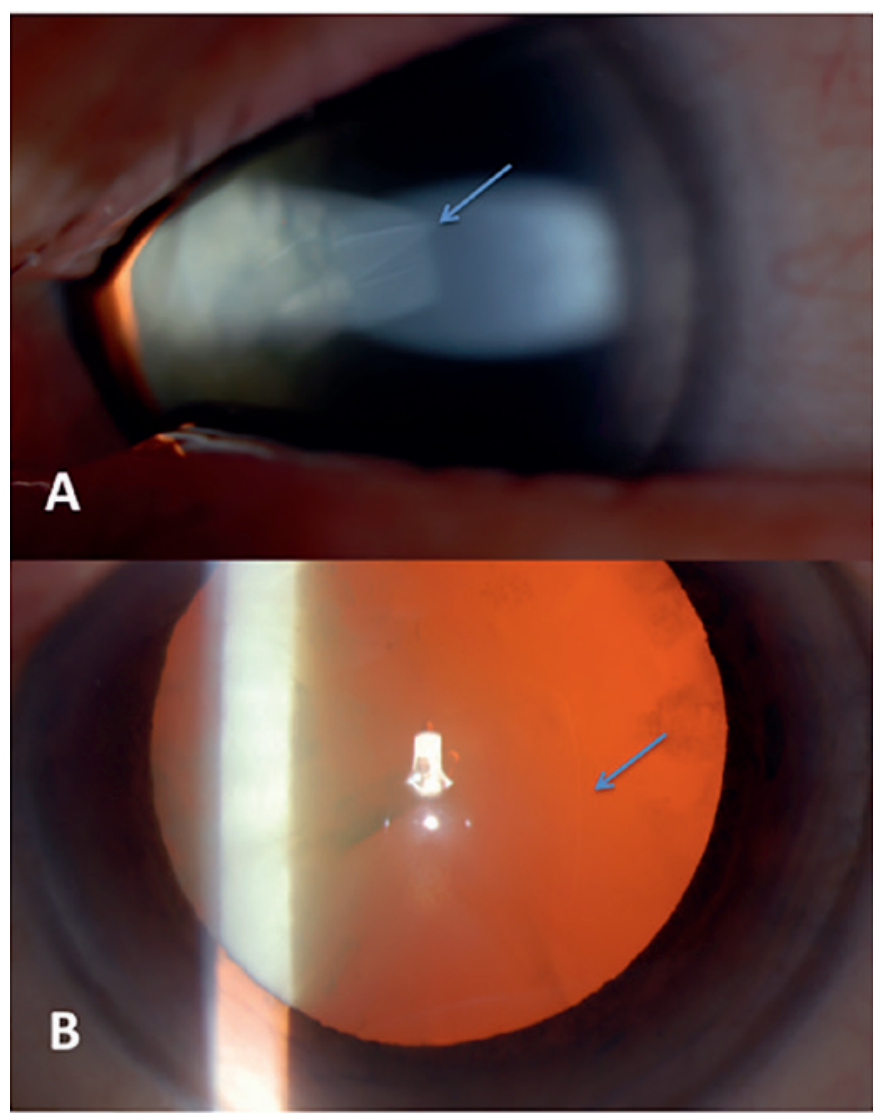

Abb. 1. a Biomikroskopisches Spaltlampenbild, das eine in dervorderen Augenkammer schwebende, abgeschilferte vordere Linsenkapsel (Pfeil) zeigt. b Der Rand der Abschilferung (Pfeil) in der Retroillumination. gische Untersuchung der Kapsulorhexis-Probe vorgenommen (Abb. 3). Bei 2 Patienten (Fälle 1 und 5) war das andere Auge pseudophak aufgrund einer früheren, in einer anderen Einrichtung durchgeführten Katarakt-Operation, sodass wir nicht feststellen konnten, ob eine echte Exfoliation vorgelegen hatte. Von den anderen 4 Patienten war die echte Exfoliation der Linsenkapsel bei einem Patienten (Fall 6) beidseitig vorhanden und bei den anderen 3 Patienten nur bei einem Auge.

\section{Fall 1}

Eine 89-jährige Frau ohne Augenerkrankungen in der Anamnese stellte sich mit Katarakten vor. Präoperativ zeigte sich in der Spaltlampen-Biomikroskopiewurde eine abgeschilferte, in der vorderen Augenkammer schwebende vordere Linsenkapsel (Abb. 1a); der Rand der Abschilferung war in der Retroillumination zu sehen (Abb. 1b). Die Patientin unterzog sich einer Phakoemulsifikation, für die eine Färbung der Kapsel mit Tryptanblau (Vision Blue, 0,1\%; DORC International BV, Zuidland, Niederlande) erforderlich war. Die Histologie der vorderen Kapsel zeigte Bereiche fokaler Spaltung (Abb. 2a).

\section{Fall 2}

Auch der zweite Patient war eine 89-jährige Frau. Präoperativ wies sie alte Hornhautnarben auf, aber laut eigenen Angaben gab es keine Augenverletzungen oder -entzündungen in der Vorgeschichte, und es konnte keine spezifische Ursache für die Hornhautnarben festgestellt werden. Die abgeschilferte vordere Linsenkapsel wurde intraoperativ bemerkt. Die Phakoemulsifikation erfolgte problemlos, ohne dass eine Kapselfärbung notwendig gewesen wäre. Postoperativ wurde festgestellt, dass die Patientin eine progressive Zunahme der Cup-Disc-Ratio aufwies, jedoch einen normalen Augeninnendruck hatte. In der Folge wurde bei ihr ein Normaldruckglaukom diagnostiziert.

Abb. 2. Mikrofotografien der echten Exfoliation der vorderen Linsenkapsel bei der Kapsulorhexis während einer Phakoemulsifikationsoperation. In beiden Bildern zeigen sich normale Linsenepithelzellen entlang der hinteren Oberfläche der vorderen Linsenkapsel. Es wurde Filterpapier verwendet, um eine flache Befestigung der Linsenkapsel zu erreichen. a Es ist eine lamellenförmige Spaltung entlang der vorderen Linsenkapsel zu sehen (HE. × 40). b Ein Aufrollen der vorderen Linsenkapsel ist festzustellen (grüner Pfeil, HE. × 20).

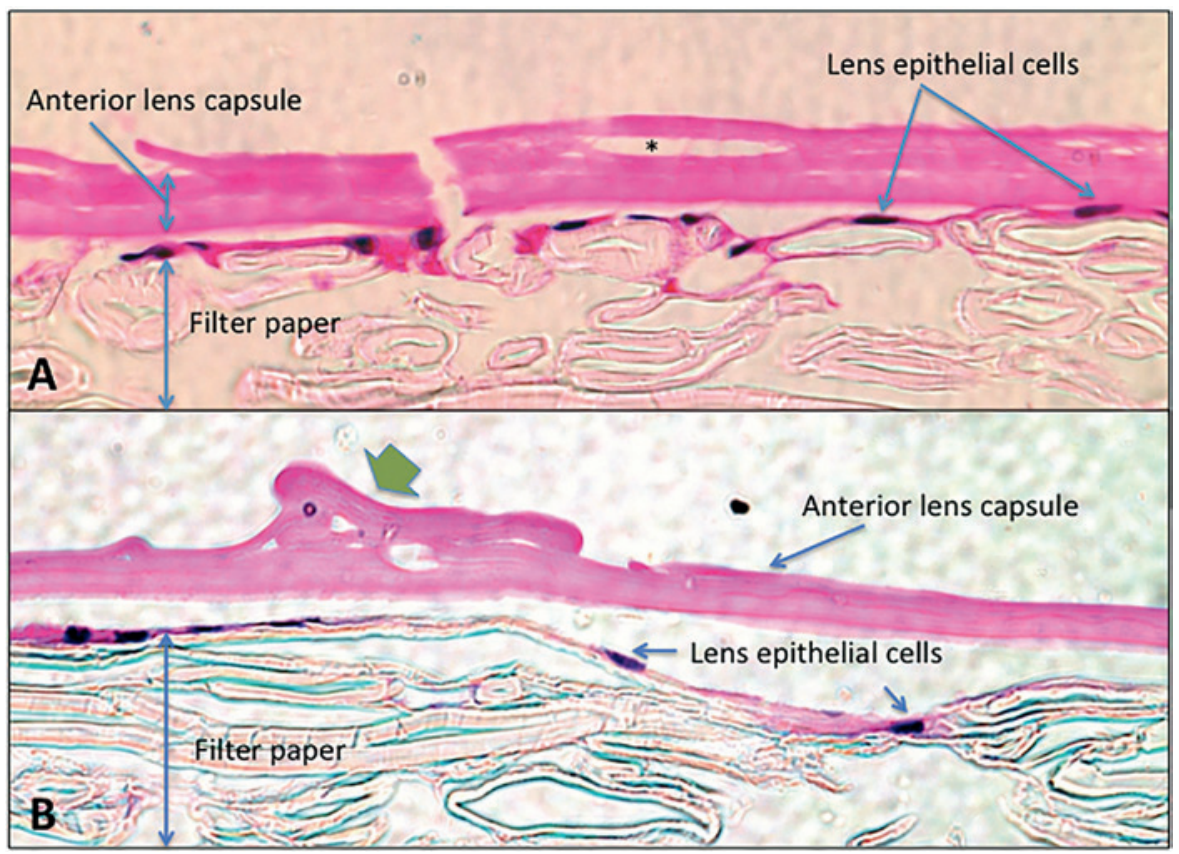


Tab. 1. Klinische Merkmale von 6 Patienten mit echter Linsenexfoliation

\begin{tabular}{|c|c|c|c|c|c|c|c|c|c|c|c|}
\hline Fall & $\begin{array}{l}\text { Alter, } \\
\text { Jahre }\end{array}$ & Geschlecht & $\begin{array}{l}\text { OD/ } \\
\text { OS }\end{array}$ & Anamnese & $\begin{array}{l}\text { Einstufung } \\
\text { der } \\
\text { Katarakt }\end{array}$ & $\begin{array}{l}\text { Linsenstatus } \\
\text { 2. Auge }\end{array}$ & $\begin{array}{l}\text { Frühere } \\
\text { Augenerkran- } \\
\text { kungen }\end{array}$ & $\begin{array}{l}\text { Präop. } \\
\text { SS }\end{array}$ & $\begin{array}{l}\text { Postop. } \\
\text { SS }\end{array}$ & $\begin{array}{l}\text { Diagnose- } \\
\text { Zeitpunkt } \\
\text { der echten } \\
\text { Exfoliation }\end{array}$ & $\begin{array}{l}\text { Intraoperative } \\
\text { Komplikatio- } \\
\text { nen und } \\
\text { Anmerkungen }\end{array}$ \\
\hline 1 & 89 & $\mathrm{~F}$ & OS & $\begin{array}{l}\text { Hypertonie, } \\
\text { Vorhof- } \\
\text { flimmern }\end{array}$ & $\mathrm{NS}+\mathrm{CC}++$ & Pseudophak & Keine & 0,3 & 0,7 & $\begin{array}{l}\text { Präoperativ } \\
\text { Bestätigt } \\
\text { durch } \\
\text { Histologie }\end{array}$ & $\begin{array}{l}\text { Keine; } \\
\text { Vision-blue- } \\
\text { unterstützte } \\
\text { Kapsulorhexis }\end{array}$ \\
\hline 2 & 89 & F & OS & Hypertonie & $\mathrm{NS}++\mathrm{CC}++$ & $\begin{array}{l}\text { Keine Linsen- } \\
\text { exfoliation }\end{array}$ & $\begin{array}{l}\text { Schwache Horn- } \\
\text { hautnarben (nach } \\
\text { eigenen Angaben } \\
\text { kein Trauma } \\
\text { vorhanden) } \\
\text { Normaldruck- } \\
\text { glaukom nach } \\
\text { Katarakt-OP } \\
\text { diagnostiziert }\end{array}$ & 0,2 & 0,5 & Intraoperativ & Keine \\
\hline 3 & 85 & M & OD & $\begin{array}{l}\text { Hypertonie, } \\
\text { Gicht, } \\
\text { ischämische } \\
\text { Herzkrank- } \\
\text { heit, Hyper- } \\
\text { lipidämie }\end{array}$ & $\mathrm{NS}++\mathrm{CC}++$ & $\begin{array}{l}\text { Keine Linsen- } \\
\text { exfoliation }\end{array}$ & Keine & 0,3 & 0,6 & Intraoperativ & Keine \\
\hline 4 & 72 & M & OS & $\begin{array}{l}\text { Hypertonie, } \\
\text { Hyperlipi- } \\
\text { dämie }\end{array}$ & NS++ & $\begin{array}{l}\text { Keine Linsen- } \\
\text { exfoliation }\end{array}$ & $\begin{array}{l}\text { Bitemporaler } \\
\text { Gesichtsfeld- } \\
\text { defekt durch } \\
\text { Hypophysen- } \\
\text { Makroadenom }\end{array}$ & 0,1 & 0,7 & Intraoperativ & Keine \\
\hline 5 & 83 & $\mathrm{~F}$ & OS & $\begin{array}{l}\text { Brust- } \\
\text { karzinom (in } \\
\text { Remission) }\end{array}$ & $\mathrm{NS}++\mathrm{CC}++$ & Pseudophak & $\begin{array}{l}\text { Akutes Eng- } \\
\text { winkelglaukom } \\
\text { in Vergangenheit } \\
\text { mit peripherer } \\
\text { Laser-lridotomie. } \\
\text { Normaler Auge- } \\
\text { ninnendruck }\end{array}$ & 0,2 & 0,7 & Präoperativ & $\begin{array}{l}\text { Keine; } \\
\text { Vision-blue- } \\
\text { unterstützte } \\
\text { Kapsulorhexis }\end{array}$ \\
\hline 6 & 92 & M & OS & Hypertonie & $\begin{array}{l}\text { NS+++- } \\
\text { PSC++ }\end{array}$ & $\begin{array}{l}\text { Echte } \\
\text { Exfoliation }\end{array}$ & Keine & 0,03 & 0,7 & $\begin{array}{l}\text { Präoperativ } \\
\text { Bestätigt } \\
\text { durch } \\
\text { Histologie }\end{array}$ & Keine \\
\hline
\end{tabular}

NS = Nukleäre Sklerose; CC = kortikale Katarakt; PSC = hintere subkapsuläre Katarakt; SS = Sehschärfe, als Dezimalzahl nach Snellen;

Präop. $=$ präoperativ $;$ Postop. $=$ postoperativ.

\section{Fall 3}

Bei einem 85-jähriger Mann mit einer beschwerdefreien ophthalmischen Vorgeschichte wurde intraoperativ eine partiell abgeschilferte vordere Linsenkapsel festgestellt. Die Phakoemulsifikation verlief komplikationslos, ohne dass eine Kapselfärbung notwendig gewesen wäre.

\section{Fall 4}

Ein 72-jähriger Mann hatte eine bitemporale Hemianopsie aufgrund eines Makroadenoms der Hypophyse. Seine Anamnese war ansonsten unauffällig. Auch in diesem Fall verlief die Phakoemulsifikation ohne Probleme und Notwendigkeit einer Kapselfärbung.

\section{Fall 5}

Eine 83-jährige Frau stellte sich mit Katarakten vor. Vor einigen Jahren erlitt sie einen akuten Engwinkelglaukom-Anfall und unterzog sich einer Laser-Iridotomie. Ihr Augeninnendruck war seit dem akuten Glaukomanfall normal und es war keine Glaukom-Medikation erforderlich. Intraoperativ wurde eine abgeschilferte vordere Linsenkapsel diagnostiziert. Auch diese Patientin unterzog sich einer Phakoemulsifikation, die ohne Komplikationen verlief. Zur entsprechenden Visualisierung der Linsenkapsel war eine Kapselfärbung erforderlich. Abbildung 3 zeigt das Doppelringzeichen, das intraoperativ festgestellt wurde. 


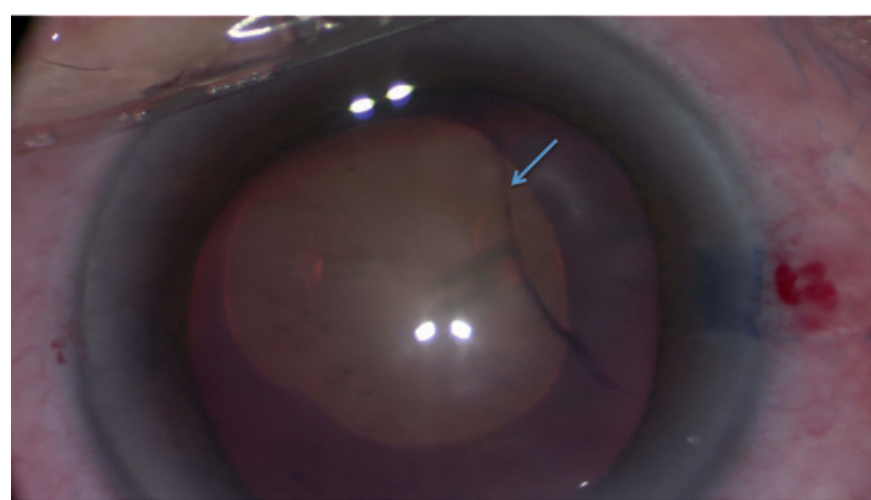

Abb. 3. Intraoperative Aufnahme, die das Doppelringzeichen bei Fall 5 zeigt. Nach Abschluss der durch Tryptanblau-Färbung unterstützten Kapsulorhexis wurde das Doppelringzeichen (Pfeil) bei einer weiteren, verbleibenden Schicht der vorderen Kapsel festgestellt. Es war eine zweite Kapsulorhexis erforderlich.

\section{Fall 6}

Ein 92-jähriger Mann ohne Augenerkrankungen in der Anamnese stellte sich mit einer dichten Katarakt vor. In beiden Augen wurden abgeschilferte vordere Linsenkapseln festgestellt. Auch hier wurde eine Phakoemulsifikation durchgeführt. Die Histologie der vorderen Kapsel bestätigte die Diagnose einer echten Exfoliation (Abb. 2b).

\section{Diskussion}

Wir berichten über eine Reihe von Patienten in fortgeschrittenem Alter mit echter Exfoliation der Linsenkapsel. Die Inzidenz in unserer monozentrischen, von einem Chirurgen betreuten Fallserie betrug 2,2\% (6 von 278 Fällen). Zur Feststellung der wahren Inzidenz dieser seltenen Erkrankung wäre eine große, prospektive Studie über einen längeren Zeitraum erforderlich. Die Erkrankung stand im Einklang mit einer altersbedingten Ätiologie, da keine anderen Ursachen, wie etwa eine frühere berufsbedingte, langanhaltende Exposition gegenüber Wärmeoder Infrarotstrahlung, festgestellt wurden. Die altersbedingte Degeneration der Linsenepithelzellen könnte die Integrität der Linsenkapsel beeinträchtigen, wodurch sich Vesikel bilden. Wenn die Vesikel verschmelzen und größere Vakuolen bilden, kann es zu einer Abschilferung des vorderen Teils der Kapsel kommen, was zu echter Exfoliation führt [4-6]. Wong et al. [2] berichteten kürzlich über eine Fallserie mit chinesischen Patienten, die ein ähnliches altersbedingten Muster echter Exfoliation aufwiesen. Die Autoren beschrieben auch eine Verbindung der Erkrankung mit dem Glaukom: 29,2 \%der Patienten wiesen ein bereits bestehendes chronisches Glaukom auf, und 20,8\% hatten sich in der Vorgeschichte einer Laser-Iridotomie unterzogen. In unserer Serie hatte eine Patientin (Fall 5) in der Vorgeschichte ein akutes Engwinkelglaukom mit anschließend durchgeführter Laser-Iridotomie.

Im Allgemeinen würde man bei einer altersbedingten Erkrankung ein beidseitiges Auftreten erwarten. Von den 4 Patienten mit einem phaken zweiten Auge war einer beidseitig betroffen, während die anderen 3 einseitig von der Erkrankung betroffen waren. Es besteht jedoch die Möglichkeit einer Asymmetrie zwischen den Papillen beider Augen. Um die Möglichkeit der subklinischen Beteiligung im anderen Auge auszuschließen, könnte die Linsenkapsel bei zukünftigen Kataraktoperationen zur histologischen Untersuchung eingeschickt werden. Die übrigen 2 Patienten hatten bei ihrer Vorstellung ein pseudophakes Partnerauge. In diesen Fällen konnte die Möglichkeit einer echten Exfoliation im Partnerauge nicht mehr überprüft werden.

Die Population war mit durchschnittlich 85 Jahren eine der bisher ältesten in der Fachliteratur erwähnten Studiengruppen. Das berichtete Alter der Patienten mit echter Exfoliation lag zwischen 80,5 und 85,4 Jahren [2, 7-9]. Das höchste Durchschnittsalter von 85,4 Jahren ist bei Cashwell et al. [7] dokumentiert, mit 11 Fällen mutmaßlich altersbedingter echter Exfoliation. Hinsichtlich der geographischen Verteilung von Fällen mit echter Exfoliation haben Autoren über Fallserien aus verschiedenen Teilen der Welt berichtet, darunter Asien, Nordamerika und Europa. Die relativ geringe Zahl der in der Literatur beschriebenen Patienten lässt kaum zu, den geographischen Einfluss auf die Entwicklung von echter Exfoliation zu untersuchen.

In unserer Fallserie wurde die Diagnose einer echten Exfoliation durch Erkennen des Doppelringzeichens gestellt und/oder durch Vorliegen einer gespaltenen oder aufgerollten vorderen Kapsel, die stark mit echter Exfoliation assoziiert war [1, 5, 6, 9-11]. Bei 2 Patienten in unserer Serie erfolgte eine histologische Bestätigung des echten Exfoliationssyndroms über die Abschilferung und Aufrollen der vorderen Kapsel. Das Doppelringzeichen war auf eine partielle Aufspaltung der vorderen Kapsel zurückzuführen, wobei zwei vollständige kontinuierliche kurvilineare Kapsulorhexis-Verfahren in einer Operation erforderlich sein können, um eine komplette Kapsulorhexis zu erreichen [8]. Ataka et al. [5] zeigten im Elektronenmikroskop, dass die vordere Linsenkapsel in Fällen mit dem Doppelringzeichen horizontale Spaltungen aufwies, mit begleitenden krankhaften Epithel-Veränderungen einschließlich vakuolenähnlicher Räume und erweiterter interzellulärer Räume, was die Beziehung zur echten Exfoliation stützt.

Das Vorliegen von echter Exfoliation der Linsenkapsel erhöht in der Regel das Risiko von Kapselkomplikationen wie radialer Erweiterung und radialen Rissen $[9,11,12]$. In unserer Fallserie hatten alle Fälle des altersbedingten echten Exfoliationssyndroms ein gutes Ergebnis bei der Phakoemulsifikation und der anschließenden Implantation der Intraokularlinse in den Kapselsack. Kulkarni et al. [13] berichteten von guten Ergebnissen hinsichtlich der Sicherheit bei der Phakoemulsifikation in 5 Fällen mit echter Exfoliation infolge von langanhaltender Hitzeexposition in der Vorgeschichte. Auch Wong et al. [2] gaben gute Ergebnisse in ihrer Serie an. Bei allen unseren Patienten wurde die CCC erfolgreich durchgeführt, wenn auch in 2 Fällen eine Kapselfärbung mit dem Farbstoff Tryptanblau erforderlich war. Mit Hilfe des Farbstoffs wurde sichergestellt, dass die CCC in voller und nicht nur in partieller Dicke erfolgte. 
Wir sind der Auffassung, dass das Erkennen der echten Exfoliation - entweder präoperativ (Aufspaltung der vorderen Kapsel in der Spaltlampen-Biomikroskopie) oder intraoperativ (Doppelringzeichen) - der Schlüssel zu unserem guten Operationsergebnis ohne Kapselkomplikationen war. Um das echte Exfoliationssyndrom präoperativ zu erkennen, können auch Bildgebungsmodalitäten wie Pentacam oder die optische Kohärenztomographie des vorderen Augenabschnitts eingesetzt werden $[6,14,15]$. Unseres Wissens wies unsere Fallserie altersbedingter echter Exfoliation einen der bislang höchsten berichteten Altersdurchschnitte auf. In allen Fällen konnte ein gutes Operationsergebnis aufgrund einer raschen Erkennung der Erkrankung erzielt werden.

\section{Ethikerklärung}

Die Patienten unterzeichneten vor der Operation eine Patienteninformation und Einwilligungserklärung.

\section{Disclosure Statement}

Die Autoren haben keine finanziellen oder proprietären Interessenkonflikte in Bezug auf die Inhalte des Artikels.

\section{Literatur}

1 Braude LS, Edward DP: Partial splitting of the anterior lens capsule giving a 'double-ring' sign. Arch Ophthalmol 1995;113:705-708.

2 Wong AL, Chan TC, Fong AH, Lam BN, Yuen HK: Clinical characteristics and surgical outcomes of phacoemulsification in true exfoliation syndrome. J Cataract Refract Surg 2014; 40:82-86.

3 Yamamoto N, Miyagawa A: True exfoliation of the lens capsule following uveitis. Graefes Arch Klin Exp Ophthalmol 2000;238:1009-1010.

4 Shentu XC, Zhu YN, Gao YH, Zhao SJ, Tang YL: Electron microscopic investigation of anterior lens capsule in an individual with true exfoliation. Int J Ophthalmol 2013;6:553-556.

5 Ataka S, Kohno T, Kurita K, Wada S, Takahashi Y, Shiraki K: Histopathological study of the anterior lens capsule with a double-ring sign. Graefes Arch Klin Exp Ophthalmol 2004;242: 245-249.
6 Cooke CA, Lum DJ, Wheeldon CE, Teoh H, McGhee CN: Surgical approach, histopathology, and pathogenesis in cataract associated with true lens exfoliation. J Cataract Refract Surg 2007;33:735-738.

7 Cashwell LF Jr, Holleman IL, Weaver RG, van Rens GH: Idiopathic true exfoliation of the lens capsule. Ophthalmology 1989;96: 348-351.

8 Kumari R, Tadros A: Double complete capsulorhexis required for cataract extraction: is it a sign of true exfoliation? Int Ophthalmol 2013; 33:285-287.

9 Yamamoto Y, Nakakuki T, Nishino K, Yagyu K, Kishi S, Ueno H, et al: Histological and clinical study of eyes with true exfoliation and a double-ring sign on the anterior lens capsule. Can J Ophthalmol 2009;44:657-662.

10 Wollensak G, Wollensak J: Double contour of the lens capsule edges after continuous curvilinear capsulorhexis. Graefes Arch Clin Exp Ophthalmol 1997;235:204-207.
11 Abe T, Hirata H, Hayasaka S: Double-ring and double-layer sign of the anterior lens capsule during cataract surgery. Jpn J Ophthalmol 2001;45:657-658.

12 Kim KH, Chung ES, Chung TY: Radial extension of capsulorhexis in true exfoliation patient: a potentially hazardous complication. J Cataract Refract Surg 2009;35:590-592.

13 Kulkarni AR, Al-Ibrahim J, Haider S, Elsherbiny S, Scott R: Phacoemulsification in true exfoliation of the lens capsule: a case series. Eye (Lond) 2007;21:835-837.

14 Chamney SM, Hughes ME, Sinton JE: The use of Pentacam in the assessment of true exfoliation of the lens capsule. Eur J Ophthalmol 2015;25:e50-e52.

15 Tan DK, Aung T, Perera SA: Novel method of assessing delamination of the anterior lens capsule using spectral-domain optical coherence tomography. Clin Ophthalmol 2012;6: 945-948. 